\title{
High-Definition Liposculpture
}

\section{Carlos Oaxaca}

Additional information is available at the end of the chapter

http://dx.doi.org/10.5772/66469

\begin{abstract}
In this chapter, we describe the author's experience with the technique, the characteristics of the ideal patients and the postoperative management of these patients to obtain positive outcomes. It is of clinical importance to underline that a good skin quality is mandatory to get the best results.

We operated on various patients between 20 and 30 years of age.

Several authors have described different techniques using different devices, but skin quality, the volume of fat to be suctioned and the surgeon's skill are critical to achieving good results.
\end{abstract}

Keywords: Liposculpture, High-definition body contour, Liposculpture small volume, minimal liposculpture

\section{History "Who does not know the history is condemned to repeat it"}

Charles H. Willi (1926) was the first surgeon to inject small quiantity of fat for aesthetic purposes. Giorgio Fischer (1975) was the first to describe the removal of fat through small incisions (5 mm) using a cannula, with an internal mechanism to cut and was called the "planotome" that was attached to a suction machine. Syringe-assisted, ultrasound-assisted, laser-assisted-, water jet-assisted, power-assisted, and percussion massage-assisted liposuction techniques have evolved since. Bircoll (1982) described the use of autologous fat from liposuction and then use it for contouring and filling defects.

Morever, the addition of stem cells to fat that has been transferred to improve fat survival is considered the newest method. We also know that cosmetic surgery is changing and improving through new instruments (machines, cannulas), as well as through new ideas as noted by several authors, and modifications of old techniques. We believe that more advances and development in the field will continue in the years to come. 


\section{Introduction}

No sculpture can carve an aesthecially and shapely body without the knowledge of its anatomy, and this is where this surgery (high-definition liposculpture) is quite amazing. Therefore, as surgeons, we must know exactly and almost in a perfect fashion, the tendons, muscles, valleys and zenith (cancavities and convexities) of every single muscle in the abdomen for it to be recreated.

Over the years, the concepts of liposculpture have changed and we know about the different techniques such as super wet and wet and dry that are used to beautify body countouring.

\section{What is High-Definition Liposculpture?}

This is an advanced body sculpting technique for the rectus abdominis and oblique muscles that can give a properly selected patient excellent results, specifically a highly sculpted, athletic body shape. Alfredo Hoyos from Colombia [1, 2] was among the first surgeons who has describedthis technique with VASER, with excellent results. Generally, patient selection or eligibility to undergo this procedure to créate a highIy defined body contour is critical. Thus, talking to the patients about the clinical requirements, and overall, being objective with the results, are essential.

Note that there are different body forms: mesomorph, ectomorph, and endomorph. Depending on the body type identified, we must find the body proportion.

However, these body types will be different between women and men, and so we must have these in mind before planning the surgery.

In the abdomen, specificall for the men, there isa tendency to gain more intra-abdominal fat in the whole abdomen, including the epigastric, mesogastryc and hipogastric areas, compared to the women because men accumulate more fat in the hipogastric area but more extra-abdominal.

The anatomical form of women (Figure 1) is rounded and different from men that is an inverted trapezoid (Figure 2. Male abdomen).

Optimal male body contouring should consider the entire torso and skin draping tightly over well-developed muscles through abdominoplasty and multiple oblique ellipses [5]. Nowadays, not just the men, but also women are seeking this kind of surgery, more to impress, especially when they are In swimsuit clothes.

Thus, to recreate this kind of abdomen, several descriptions on the techniques: VASER, laser, and standard Liposuction have been published.

When there is skin excess, this will have to be removed horizontally to have the skin tightly bound to the muscle. In this case, the surgery must be done in two stages.

How will the best results be obtained? Is it true that expensive devices are needed to perform it?

The answer is "not always," as has been described and experienced by some surgeons from the USA. 


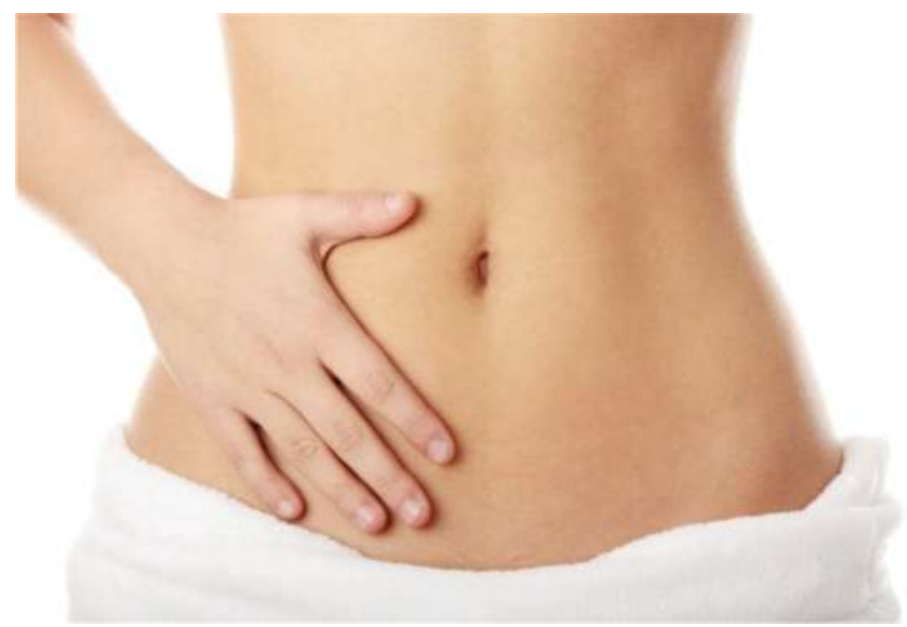

Figure 1. Female Abdomen.

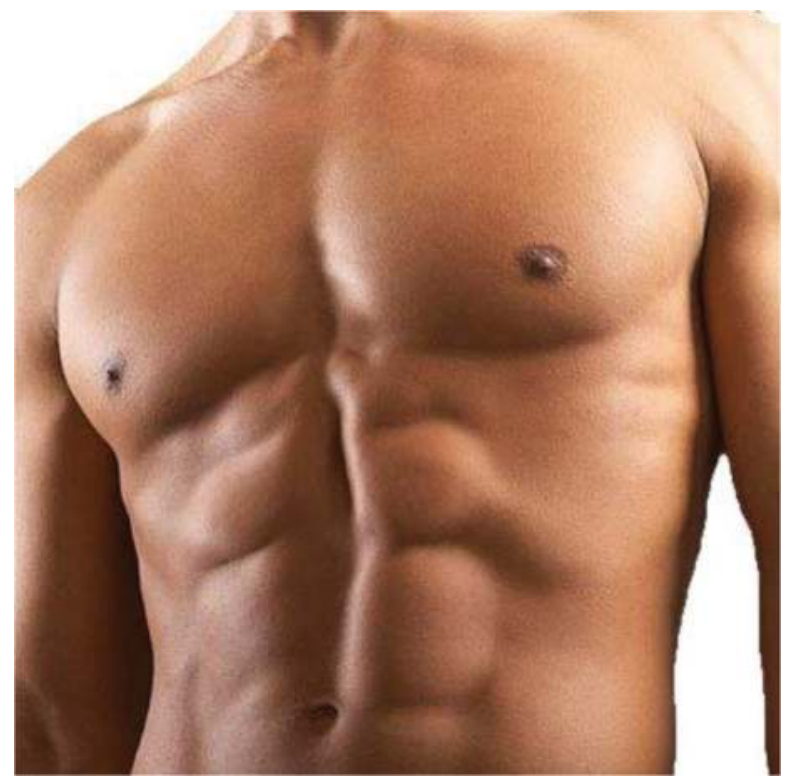

Figure 2. Male Abdomen.

It is important to mention that for best results, a good skin quality is mandatory because flaccidity will not give us high definition. In consultations with the patient, male or female, their expatatives are asked. 


\section{Anatomy}

We must know about the ideal anatomy of the abdomen:

\subsection{Rectus Abdominis}

This muscle arises from the symphysis, crest, and pecten of the pubis and runs upward to insert into the xiphoid process and costal cartilages of the fifth to seventh ribs. Its main action is to flex the trunk (Figure 3).

\subsection{External Oblique}

This muscle forms the lateral abdomen. It originates from the external and inferior surfaces of the lower seventh to eighth ribs. The external oblique has two parts: an upper thoracic portion and a lower flank portion.

\subsection{Serratus Anterior}

This quadrilateral muscle originates anteriorly as fingerlike bundles from the external surfaces of the upper eighth to ninth ribs and wraps around the rib cage to insert into the vertical, medial edge of the scapula. It acts to draw the scapula laterally and around the rib cage, as in punching.

The below parts are not in the abdomen but form part of the aesthetic anatomy of the anterior chest:

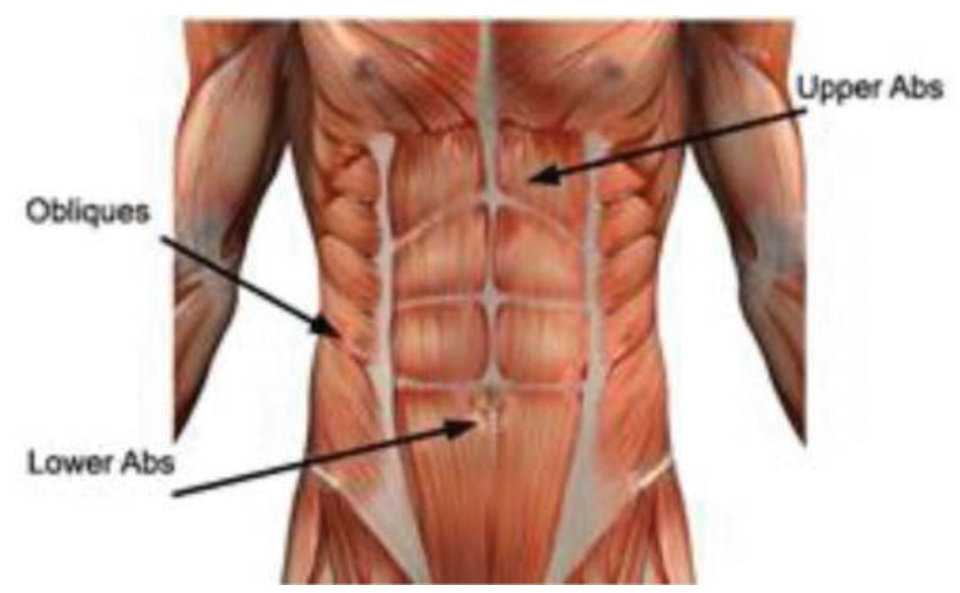

Figure 3. Anatomy of the Abdomen. 


\subsection{Pectoralis Major}

The pectoralis major forms most of the muscle bulk of the chest and gives the chest a smooth, convex form, particularly when the muscle is well developed and defined. It originates from the medial half of the clavicle, anterior surface of the sternum, costal cartilages of the first 6th-7th ribs, and inferiorly, the superior part of the external oblique aponeurosis of the abdomen.

\subsection{Latissimus Dorsi}

The latissimus dorsi is a triangular muscle that passes from the midline to the arm like a cape, covering the deeper muscles of the mid and lower back. It originates from the spinous processes of the seventh to twelfth thoracic vertebrae, those of all of the lumbar and sacral vertebrae, the posterior one-third of the iliac crest, and the external surfaces of the lower three ribs.

\section{Fat Metabolism}

Fatty tissue represents at birth, about $17 \%$ of bodyweight;and in adolescence, around $20 \%$ in women and $10 \%$ in men with some phenotypic variations. In normal adults, fat approaches 15-20\%in men and 25-30\% in women. Two types of fat are described in the human body: white adipose tissue and brown adipose tissue.Fatty tissue also plays an important role as an energy source during exercise. Fat, the major energy stored in the body, is mobilized from adipose tissue as free fatty acids to provide metabolicfuel. At lower exercise intensities, fats may provide 50-60\% of the energy for muscle contraction, but this process cannot keep pace withthe high demands for energy that occurs during heavy exercise. Energy liberation from fat is slower than the liberation of glucose from glycogen.Moderate activity, in fact, favors the consumption of fat as muscle fuel. The depletion of body fat reserves is almost never a limiting factorin muscle activity. In the absence of other fuels, proteins can serve as an energy source for contraction, particularly as it occurs during heavy, prolonged, or intense exercise.

\section{Anatomy of the Fatty Tissue}

We must consider anatomy before liposculpture can be done. We have the skin, the superficial and deep fat layer, divided by the superficial fascia (Scarpa) and the deep fascia (Camper).

Additionally, the fat thickness in the body varies. The superficial adipose tissue in the abdomen is thicker and in the gluteal and thigh, it is thinner. In the leg, there is only superficial and no deep adipose tissue.

It is extremely important to consider the fat percentage between women and men, and for the purpose of this chapter, note always that the less the fat percentage, the better the result will be (Figure 4). 


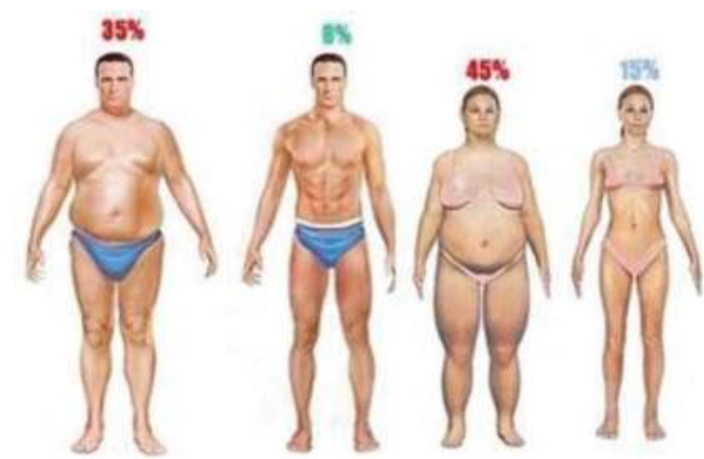

Figure. 4. Body differences between women and men.

\begin{tabular}{lll}
\multicolumn{3}{c}{ Body Fat Percentages } \\
\hline Description & Men & Women \\
Essential fat & $2-5 \%$ & $10-13 \%$ \\
Athletes & $6-13 \%$ & $14-20 \%$ \\
Fitness & $14-17 \%$ & $21-24 \%$ \\
Acceptable & $18-24 \%$ & $25-31 \%$ \\
Obese & $25 \%+$ & $32 \%+$ \\
* American Coundil on Exercise Guidelines
\end{tabular}

\section{Who is the Ideal Patient?}

The medical history of each patient is essential. Inquiring about patient intake of salicilates or other medicines is also important. Most importantly, regardless if laboratory test results are normal, we need to ask and verify if they are taking dietary complements, such as vitamin E, vitamin A, or artichoke, green tea, ginko and other similar complements that extend coagulation time.

\subsection{Indications}

The most critical factors in selecting patients are the following:

1. Skin elasticity.

2. Work up in the gym (for better results).

3. Age variability.

Patients with poor skin quality and strias are not ideal candidates for this procedure because their skin will not shrink appropriately.

Patients whose work up on gym or regularly exercising on daily basis, are preferred, because are expected that there will be less amount of fat to be suctioned, so after surgery will result in high definition muscle.

\subsection{Contraindications}

Age is an important factor with older age, there is more flaccidity. Patients who are obese are also not good candidates for this procedure.

Ahigh tendency to gain weight is also a factor.

Patients have to be analyzed in standing and sitting positions to check if muscle diastasis of the rectus abdominis muscle exists. 
Grasping the skin to look for flaccidity that could be an important factor to get the best results is also important.

Having photographs of the patient are likewise essential. First, these will help in supporting comments and observations during consultation.

Second, this could help to protect us, because many times, patients will appreciate better the result, when they see their former physical body.

That is why we always take photos pre and postoperatively and dispaly them on a big screen, after every consultation. This will avoid any claim.

\section{Preparation of the Patient}

In consultation, the availability and analysis of the patients' lab tests incluiding Haematic counts, protein levels, partial thromboplastin time and prothrombin time, and glucose, urea and creatinin levels are necessary.

The protein level is a prognostic factor to determine if there is alowriskoflung thromboembolism.

It is equally important that the patient read and sign an informed consent form where details including the type of anesthesia, the kind of the surgical procedure, and even possible complications, such as allergy, bleeding, infection, pain, pulmonary embolism, scarring, and seroma, are explained.

\section{Anesthesia}

Although there are two options for anesthesia, general anesthesia is preferred. For this type of surgery, this is not a problem because the amount of aspiration is about $600 \mathrm{ml}$ on average, but the operative time is long. Intraoperative tumescent anesthesia, using the Klein solution is as follows:

- Saline solution, $1 \mathrm{lt}$

- Sodium bicarbonate, $10 \mathrm{ml}$

- Lidocaine $2 \%, 50 \mathrm{ml}$

- Epinephrine, $1 \mathrm{ml}$

As the total aspirated volume is less than 600 to $800 \mathrm{ml}$, then we use the same amount of volume. This will decrease the bleeding.

Remember that lidocaine has two effects: create vasoconstricticon to lessen the bleeding and slow the systemic absorption of lidocaine. 
It is crucial that the surgeon always verifies the prepared solution, to avoid, in some occasions, potential mistakes from nursing staff when they prepare the solution.

\section{Markings}

The markings are the most important factor because we delineate the belly of the rectus abdominis muscle and the oblique muscles to be tailored (Figure 5).

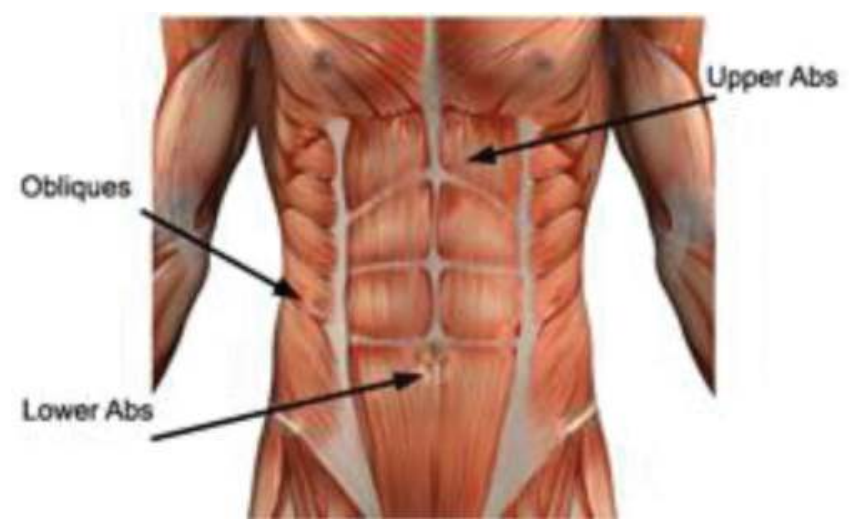

Fig. 5. Valleys and peaks to be mimicked during the liposculpture.

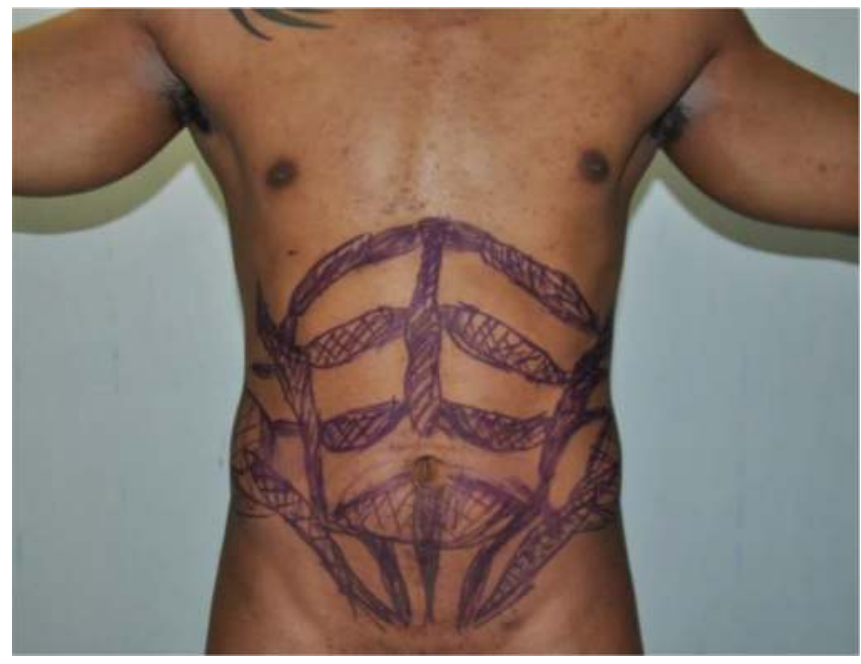

Fig. 6. Marking the patient. 
The patient must be marked in a standing position and this will take several minutes, but this is one of the most crucial parts of the procedure (Figure 6). The muscular anatomy must be recreated with the surgery and here, the valleys and peaks must be done with the cannula. This procedure takes more tan 30 minutes. An artistic drawing on the patient's body is done before the surgery in standing position.

\section{Technique}

The patient is always operated on under general anhestesia. In a standing position, the area is cleaned with betadyne and the surgical table is covered with sterilized clothing.

Saline solution (one liter), epinephrine (one ampule) and sodium bicarbonate (one) are added to infiltrate the area to be suctioned. We won't need more than $600 \mathrm{ml}$ of this solution.

Small incisions are placed as marked in Figure 6.

The male patients are completely marked because they request for a "six-pack" abdomen. Sometimes we have to define the pectoral area and this can be done by high-definition liposculpture or adding pectoral implants [4].

The treatment for women is different because they want the medial depression of the alba line to be recreated and that the demarcation between the oblique and rectus muscle (semilunar line) be defined. These are important points to be remembered or considered [4].

Another point is thinking about the intra-abdominal and extra-abdominal fat, with the former being more challenging.

Liposculpture is done with a 20-ml syringe and a 2- or 3-mm cannula. It takes about 4 hours on average and the tip of the cannula is turning up to the skin to create fibrosis from the underside of the skin to the fascia. An aggressive cannula is used like a chisel in the subcutaneous tissue for superficial liposuction, removing the fat at the level of the tendinous inscriptions, the anatomic lines, and the muscular borders, toemphasize the muscle bulk, which means recreating muscle valleys and peaks as mentioned previously.

Patients who used to be obese could be required to undergo two stages of surgery, one to excise the abdominal skin excess and then do liposuction, and the second to sculpt the abdominal muscles [4].

The critical point in attaining high definition is to highlight adherence in the tendinuous area.

The incisions are placed in the suprapubic area, generally two of 2-mm in length, another one in the umbilicus and epigastric area. Sometimes it may be required in the lateral area of the abdomen to recreate the tendinous inscriptions of the muscles. The cannulas used are 6 hole with 2- to 4-mm diameter, generally with a 20-ml syringe. If an area has more fat deposit, then a $5 \mathrm{~mm}$ diameter cannula is used, with $50 \mathrm{ml}$ siringe. Then the final touch is done with $2 \mathrm{~mm}$ cannula. 
The infiltration is done by areas, because it is time-consuming procedure, that takes more tan 4 hours, on average. Normally, infiltration is started from the hypogastric area and flanks, going superiorly, and then going from the epigastric incision and lateral incisions to create the oblique and six-pack abdomen desired by the patient. Sometimes micro-fat grafting may be required to get the best results.

Note that it is important not to simply think about creating a symmetrical "six-pack abdomen, as doing it so symmetrical, may lead to an unnatural result.

The incisions are sutured with nylon 6-0 or subcutaneous monocryl 5-0. Then a special garment is used, but in the area where surgery was performed, a foam tapped with a 1-inch wide micropore will be added and left there for at least 1 week. The garment will be retained for one month all day long and for the second month, it will be just be during the day, and then resting by night. Care must be taken not to use tied garment if micro-fat grafting has been used, otherwise it will reduce the viability of the grafted fat.

Moisturizing the skin at this moment is mandatory.

Drains are never used for these patients because the amount suctioned

\section{Postoperative Care}

The author used levofloxacine $500 \mathrm{mg}$ daily for 1 week and ibuprofen $400 \mathrm{mg}$ twice daily for 1 week. For a small amount of fat suctioned, enoxaparine is prescribed, due to the length of time of surgery.

The goal in the postoperative period is to reduce bruising, avoid hypertrophic scars and allow adhesions where there must be retraction in the tendinuous areas of the muscles, therefore, postoperative ultrasound must be avoided.

Arnica is used one tablet twice a day for 2 weeks to reduce bruising.

Manual lymphatic drainage (MLD) [3] pioneered by Emil Vodder in 1930's is done after the first postoperative week, and this will reduce postoperative edema. The strategically induced change in pressure in the subcutaneous tissues enhances filling and emptying of initial lymphatics, increases the rate of contraction of lymphatics, and increases lymphatic transport capacity. Firmer, deeper movements may be employed in localized areas to soften hardened tissue due to fibrosis or fibrosclerosis, but excessively deep, diffuse pressure is avoided to minimize capillary filtration. The benefits of manual lymphatic drainage are stimulation of the lymphatic flow and circulation; stimulation of the immune system, parasympathetic nervous system with relaxation and anti-spastic effects, and as perineural edema is lessened, pain is also reduced; fibrosis reduction, improvement of cellular nutrition; and tissue recovery. The frequency of each session must be 2 days each for at least 2 weeks. The duration of each session must be anywhere from 40 to 60 minutes. Care must be taken to ensure that MLD is carried out gently and without pain to the patient.

The stitches are taken out on the at 7th day, postoperatively, but if the suture material used is monocryl, then this will no longer be necessary. 

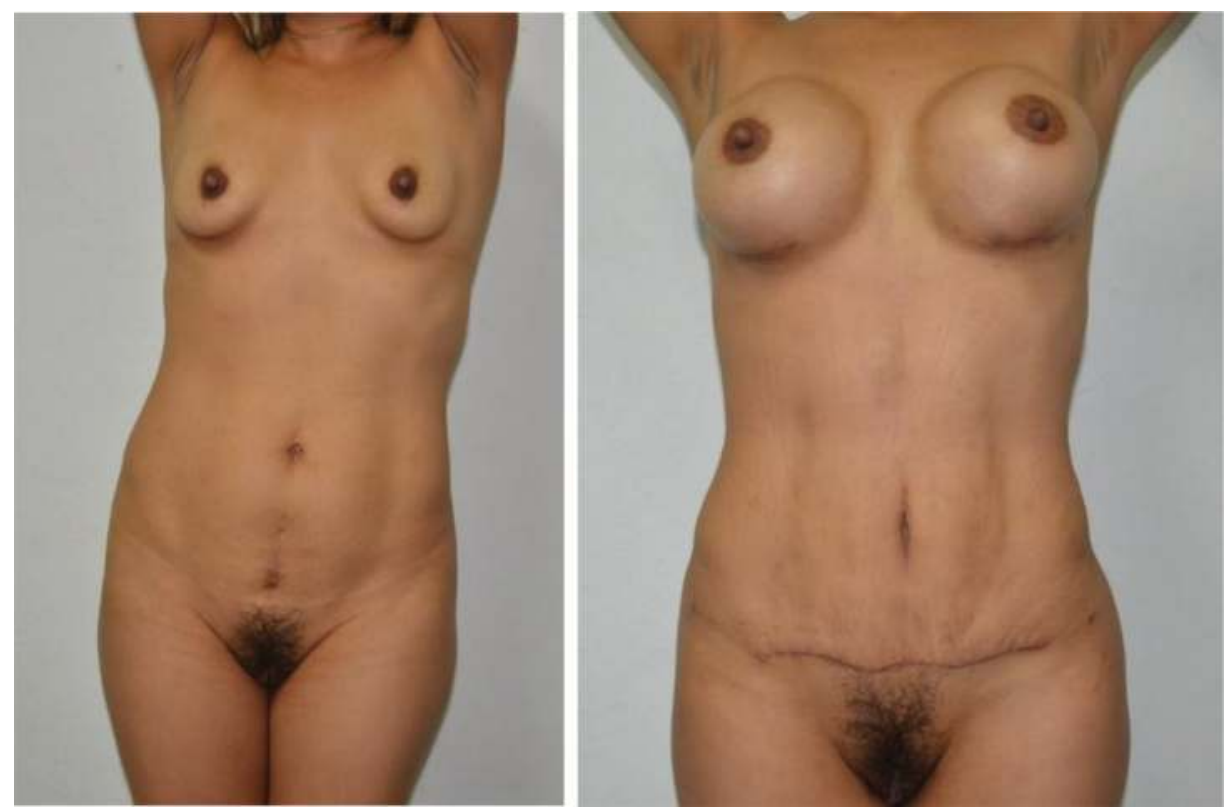

Figure 7. Frontal pre and postoperative photos: female patient with miniabdominoplasty.

Garments are used day and night for the first month postoperatively, and taken out only for cleansing. For the second month, garments are used just during the day, and taken out by night. Epifoam is used in the areas where depressions have been created in the tendinuous areas, for 3 weeks and over the garments.

\section{Starting activities}

The patients are asked to walk the next postoperative day using anti-thromboembolism socks for 1 week.

\section{Results}

The best results are obtained between 3 and 6 months postoperatively. This is always mentioned to the patients because they ask in the first postoperative consultations about the results, and they are eager to see the final result in the first month. The appointments are made in the office at Day 7 and then 1, 3, 6 and 12 months postoperatively. At 3rd month, (before and after) photos are taken and shown to the patient on a big screen, this way, they will see the results and feel more comfortable about the outcome. Results for the female patients are shown in Figures. 7, 8, and 9. Results for the male patients are shown in Figures 10, and 11. 

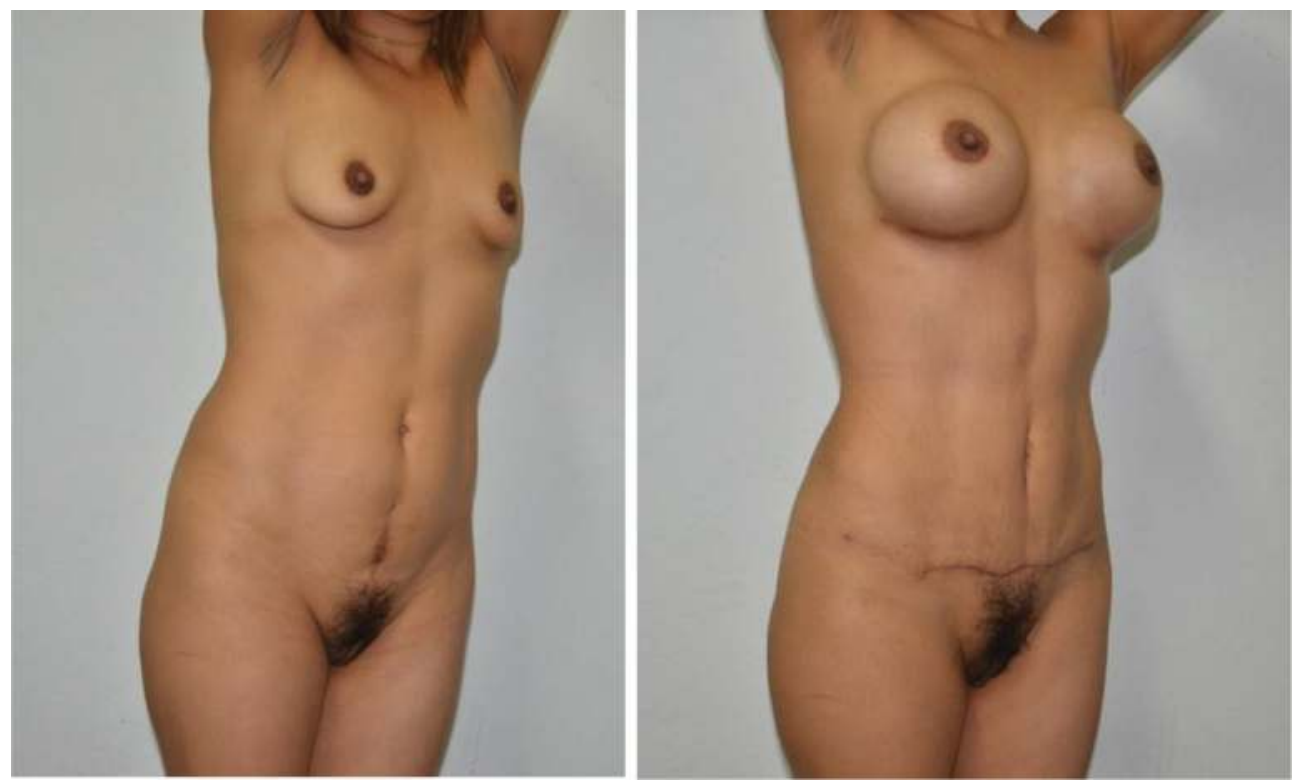

Figure 8. Pre and postoperative photos, right oblique on a female patient.
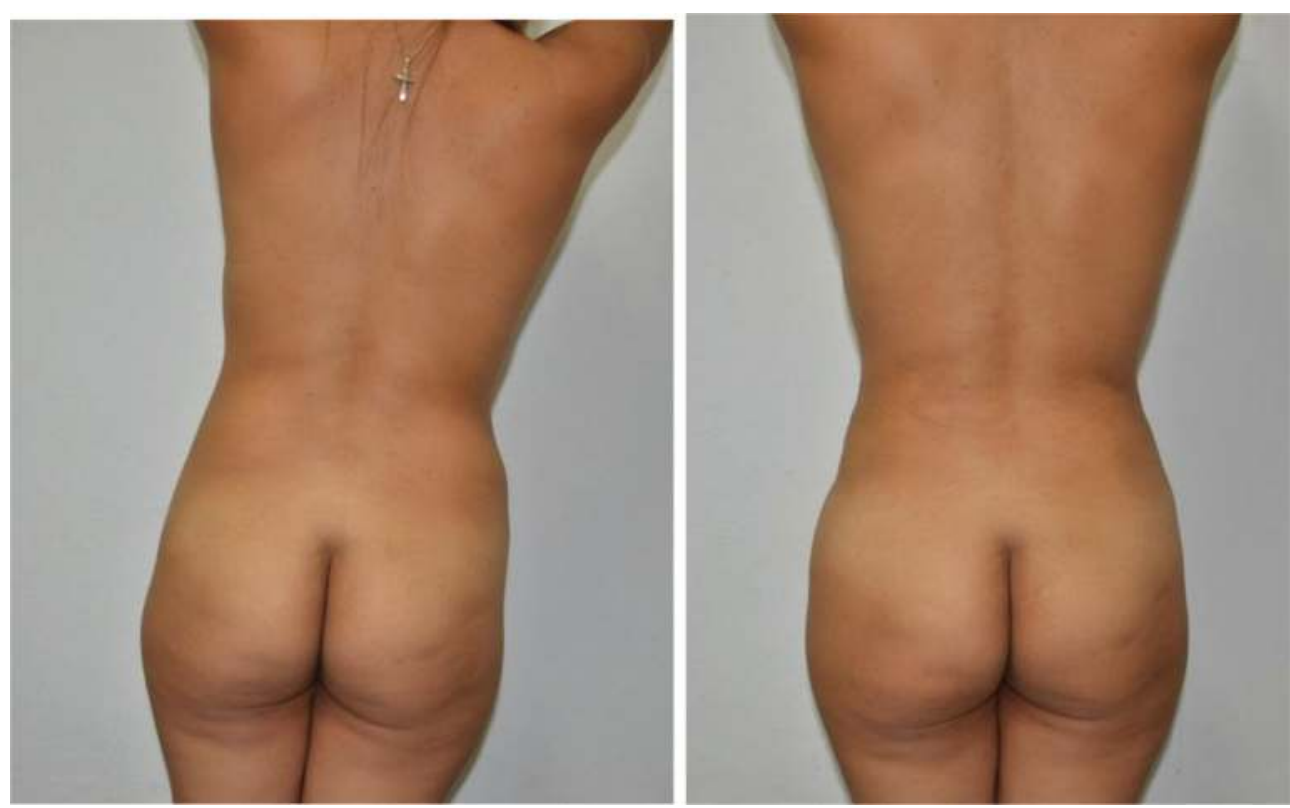

Figure 9. Pre and postoperative photos: female patient. 

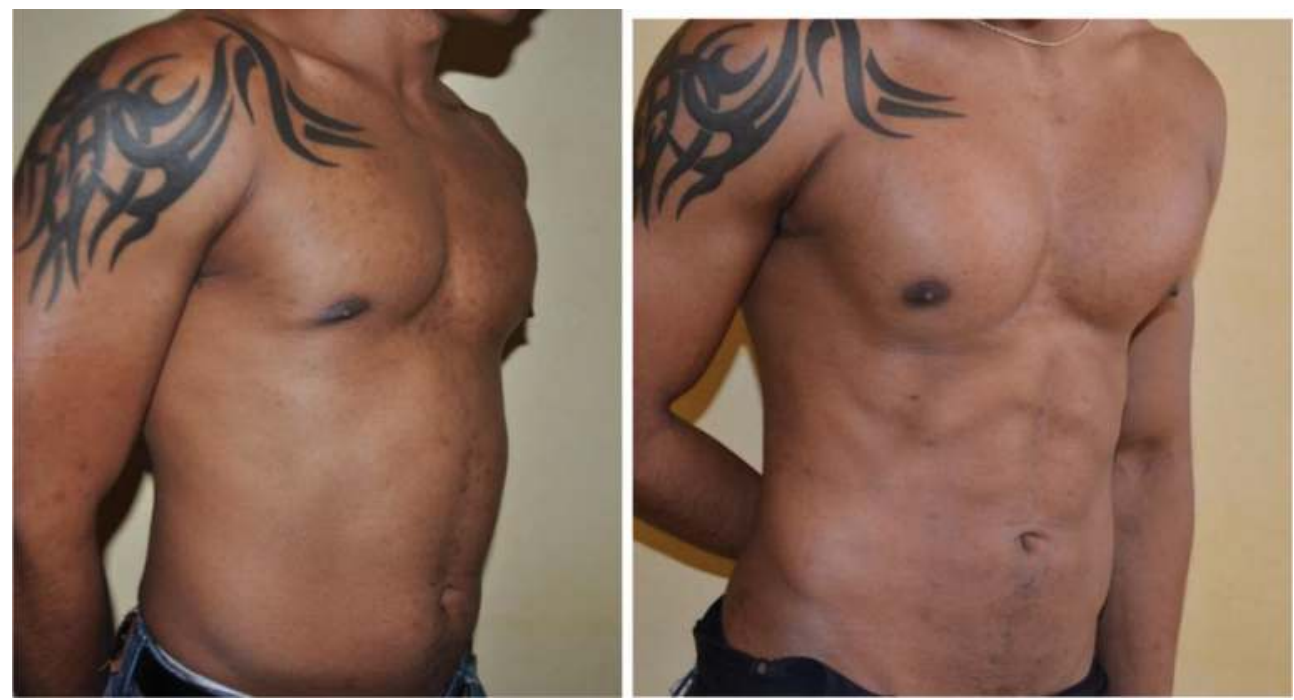

Figure 10. Right oblique pre and postoperative on a male patient.
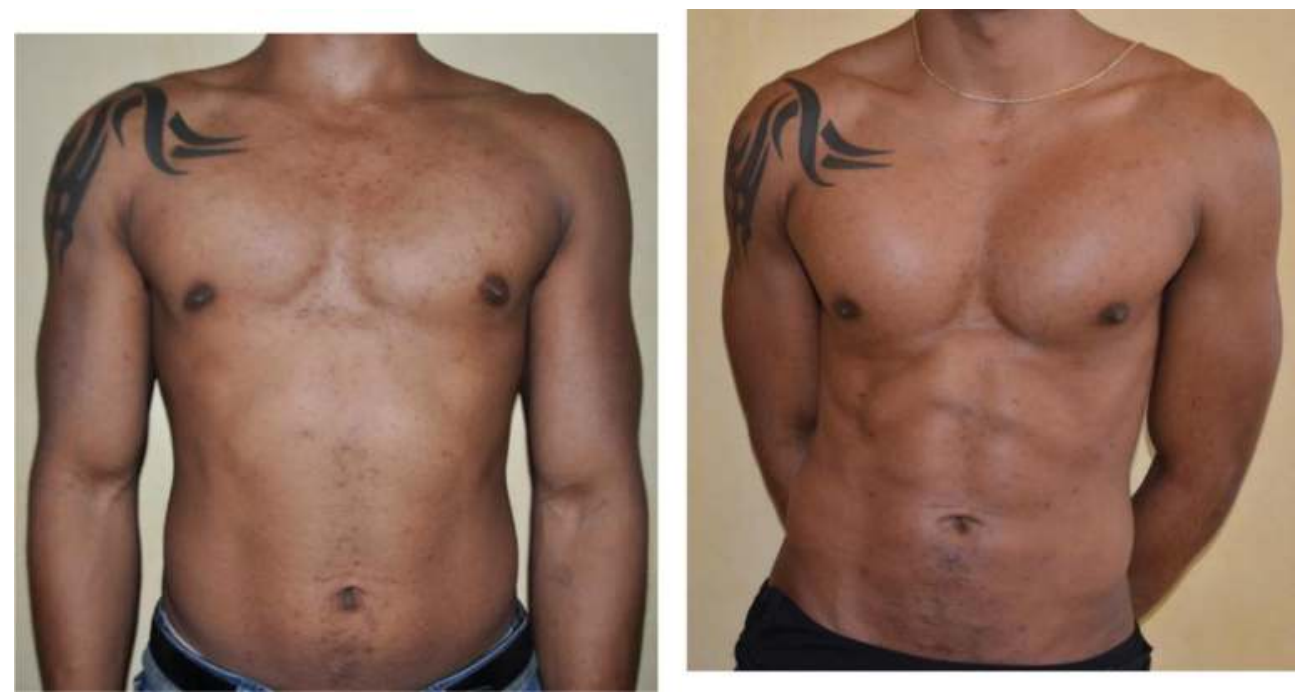

Figure 11. Frontal pre and postoperative photos on a male patient: mild assymetry on the abdomen that looks more natural. 


\section{Complications}

It is very seldom to have complications for this surgery,given that patients are well-evaluated during preoperative stage. Bleeding is also rare as per the author's experience.

Infection may be a possibility, and so antibiotics should be started 30 minutes prior to surgery and continuing for 7 days in the postoperative period.

Skin necrosis is a high possibility with aggressive suction with the cannula when it is turned against the skin.

Seroma, pulmonary edema, thromboembolism and perforation are another complications reported, but these may be reduced, if not eliminated, with careful administration of the technique, given that it is superficial; Pinching the skin all the time, with the other hand, while cannula is passing under the skin, is necessary and helpful.

Lidocaine toxicity is rare because the amount of fluid infiltrated in the areas to be treated is very small. On large liposuctions, this complication can occur, but now, the author, has abandon its use, because the patient is under general anesthesia, then lidocaine is no longer required and this will reduce chances of toxicity.

Burns on the skin incisions because of friction caused by cannulas going in and out of the skin is a high possibility. Instiling water over the entrance of the incisions is recommended.

The absence of depressions in the sculpted areas after the surgery could be considered as a complication, but well-selected patients do not develop it. Skin retractions on sites that are not planned could happen sometimes when the cannula shifts during suctioning.

Skin flaccidity is a more common complication when patients are not selected properly.

Another potential and critical complication that must be shared to the patients is that if they gain weight and become fat in the future, the appearance of their abdomen may be likened to that of a turtle, but with a "six pack". Therefore, it is important to highlight to patients the need to always be in check of their weight.

\section{Discussion}

High-definition liposculpture is a highly demanding surgical procedure that needs the expertise of plastic surgeons who perform this procedure regularly. As always, the expertise is mandatory to get the best results.

Having experienced plastic surgeons and the accurate analysis as well as adequate selection of patients are crucial because these will impact having satisfied patients.

Always remember that the final result will be seen after 3 months, generally, and this must be mentioned to the patiens. 


\section{Conclusion}

We have presented the critical points in this chapter, beginning with the anatomy, preoperative markings, standardized photos, and how the procedure must be done, apart from the ideal cannulas to be used. The purpose of this chapter is to establish the critical points to ensure a successful surgery.

\section{Author details}

Carlos Oaxaca

Address all correspondence to:droaxaca@hotmail.com

Plastic Surgeon, Torre Medica Santé, Leon, Guanajuato, México

\section{References}

[1] Hoyos E. Alfredo, Millard John. VASER assisted High Definition Liposculpture. Scientific Forum, 2007, Nov 27 (6) 594-604.

[2] Hoyos E. Alfredo, Perez Mauricio. Arm Dynamic Definition by Liposculpture and Fat Grafting. Aesthetic Surg J. 2012 32(8) 974-987.

[3] Hoyos Alfredo, Prendergast Peter. High Definition Body Sculpting. Springer Editorial, 2014.

[4] Yi Xin Zhang, David Lazzer, et al. Three-Dimensional Superficial Liposculpture of the Hips, Flanks and Thighs. Plast Reconstr Surg Global Open, 2015; 3: e291.

[5] C. Monarca, M. I. Rizzo. New Body Contouring Technique in Male: The Muscular Sculpture Aesth Plast Surg (2015) 39: 199-202. 
